\title{
Stability and Equilibrium States of Infinite Classical Systems
}

\author{
Michael Aizenman ${ }^{\star}$ \\ Princeton University, Department of Physics and Mathematics, Princeton, N.J., USA \\ Giovanni Gallavotti $\star \star$ \\ Istituto Matematico, University di Roma, I-00185 Roma, Italy

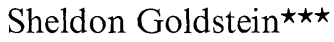 \\ Cornell University, Department of Mathematics, Ithaca, N.Y., USA \\ Joel L. Lebowitz ${ }^{\star \star}$ \\ Belfer Graduate School of Science, Yeshiva University, New York, N.Y. 10033, USA
}

\begin{abstract}
We prove that any stationary state describing an infinite classical system which is "stable" under local perturbations (and possesses some strong time clustering properties) must satisfy the "classical" KMS condition. (This in turn implies, quite generally, that it is a Gibbs state.) Similar results have been proven previously for quantum systems by Haag et al. and for finite classical systems by Lebowitz et al.
\end{abstract}

\section{Introduction}

It is generally accepted that the appropriate microscopic description of both equilibrium and nonequilibrium properties of bulk matter is via "macroscopic states". These states are (when quantum effects are unimportant) probability measures on the phase space $K$ of an infinite system of indistinguishable particles moving in $\mathbb{R}^{3}[1,2]$. When the system is in equilibrium it is assumed that the appropriate macroscopic state is a Gibbs (or equilibrium) measure at some temperature $\beta^{-1}$ and fugacity $z$. There are several alternative ways of describing these measures, e.g. the DLR equations, the Kirkwood-Salsburg equations, etc. These are however all essentially equivalent $[3,4]$ : the infinite volume Gibbs states corresponding (as they presumably should physically) to an appropriate limit of finite volume grand canonical ensembles. Other finite volume ensembles such as the microcanonical and the canonical are also expected to have Gibbs states as limits [5].

In this paper we investigate the problem of justifying the use of Gibbs measures for infinite classical systems. (The analogous problem for finite classical system was treated in [6].) Of course, any measure $\omega$ which describes the state of a system in equilibrium must be stationary, i.e. invariant under the time evolu-

\footnotetext{
* Supported by N.S.F. Grant MPS 71-03375 A03. Part of this work was carried out at the Courant Institute where it was supported by N.S.F. Grant GP-37069X.

$\star \star$ Supported in part by AFOSR Grant \# 73-2430 and N.S.F. Grant MPS75-20638.

$\star \star \star$ Supported by N.S.F. Grant \# GP33136X-2. Part of this work was carried out at the Institute for Advanced Study.
} 
tion $\left(\alpha_{t}^{H} \equiv \alpha_{t}\right)$ generated by the system's Hamiltonian $H: \omega(f) \equiv \int f d \omega=\int \alpha_{t} f d \omega$. The existence of such an evolution and the invariance of Gibbs measures under its action has been proven recently by Lanford [7,2], Sinai [8], and by Marchioro et al. [9]. However, the Gibbs states may not be the only stationary, invariant measures. Even the requirement that the measures which are to describe macroscopic states be sufficiently "regular" may not rule out extra stationary states [10]. Thus in the ideal gas system there exist stationary states corresponding to any given velocity distribution, not only the Maxwellian. It is therefore of interest to see whether the imposition of additional physical requirements on the measures appropriate for the description of equilibrium systems will single out the Gibbs measures.

One such suitable physical requirement is provided by consideration of a stability condition similar to the one introduced by Haag, Kastler and TrychPohlmeyer (HK T-P) [11] for quantum systems. (Indeed, their work, which has since been extended further [12-14] was the motivation for this investigation and our analysis and results are closely related to theirs.) This condition may be motivated by the following consideration: We can never know precisely the exact nature of the forces under which our system evolves. Therefore, if near a state $\omega$ stationary for $\alpha_{t}$ there were no state stationary under a time evolution obtained by a "small local perturbation" of $H, \omega$ could not be regarded as physically reasonable. We may therefore require that, for small $\lambda$, there exists a state $\omega^{\lambda h}$ stationary for the time evolution given by the Hamiltonian $H+\lambda h$ such that $\omega^{\lambda h} \rightarrow \omega$ as $\lambda \rightarrow 0$. As we shall see such a requirement of stability on the state $\omega$ imposes non-trivial constraints on the form of $\omega$ which tend to single out the Gibbs states. The Gibbs states $\omega$ are stable since the state $e^{-\beta \lambda h} \omega / \omega\left(e^{-\beta \lambda h}\right)$ is stationary for $H+\lambda h$.

To obtain a precise formulation of stability we need to specify the class of perturbations $h$ under which $\omega$ is required to be stable as well as the manner in which $\omega^{\lambda h}$ is to approach $\omega$ as $\lambda \rightarrow 0$. Since our use of the measure $\omega$ is to obtain expectation values of "physical" observables closeness should refer to such expectation values, i.e. we should require at the minimum that as $\lambda \rightarrow 0, \omega^{\lambda h}(f) \rightarrow \omega(f)$ for such observables. Having said this much there does not seem to be any unique way to be more specific. We would naturally like to make the assumptions as weak as possible and the conclusions as strong as possible. For the purpose of this paper it is technically convenient to assume that stability under the perturbation $h$ requires that for $\lambda \in[0, \delta], \delta>0, \omega^{\lambda h}$ may be chosen to be given by an $L^{2}(\omega)$ density function. A state $\omega$ is then called stable only if $\omega^{\lambda h}(f) \rightarrow \omega(f) \forall f \in L^{2}(\omega)$ and all $h$ belonging to some class $\mathscr{D}$ which will be specified later.

We prove that stability plus some strong clustering conditions imply that $\omega$ satisfies the classical analogue [15] of the quantum KMS condition. Let

$$
F_{f, g}(t)=\omega^{T}\left(f, \alpha_{t} g\right)=\omega\left(f \alpha_{t} g\right)-\omega(f) \omega(g)
$$

and let

$$
G_{f, g}(t)=\omega\left(\left\{f, \alpha_{t} g\right\}\right)
$$

where $\{f, g\}$ is the Poisson bracket between $f$ and $g$ (see Def. 1). Then the classical 
KMS condition, in its dynamical form $\left(\mathrm{KMS}_{\mathrm{D}}\right)$, says that

$$
G_{f, g}(t)=-\beta \frac{d}{d t} F_{f, g}(t), \quad \forall f, g \in \mathscr{D}
$$

with $\beta$ some constant. For the cases we are considering $\mathrm{KMS}_{\mathrm{D}}$ implies an equilibrium $\mathrm{KMS}$ condition $\left(\mathrm{KMS}_{\mathrm{E}}\right)$, not involving the time evolution:

$$
\omega(\{f, g\})=\beta \omega(g\{f, H\}) .
$$

It can be shown $[15,16]$ that when $\mathscr{D}$ is "large enough" (1.4) implies (using some additional mild assumptions) that $\omega$ is a Gibbs state. (It may be readily seen that the finite volume canonical ensemble, $d \omega=(1 / Z) e^{-\beta H} \prod_{i} d q_{i} d p_{i}$, satisfies the $\mathrm{KMS}_{\mathrm{E}}$ condition for functions with proper boundary conditions.) It is the balance between desiring to make $\mathscr{D}$ small so as to minimize the assumptions and the requirement that $\mathscr{D}$ be large enough so that $\mathrm{KMS}_{\mathrm{E}} \Rightarrow$ Gibbs that determines the choice of $\mathscr{D}$.

The argument which we give is, for the most part, related to that of HKT-P (avoiding however the use of Fourier representation for the $\mathrm{KMS}_{\mathrm{D}}$ condition) with the proper replacement of commutators by Poisson brackets (PB). Unfortunately the $\mathrm{PB}$, involving derivatives, is not as nice mathematically as the commutator. In particular $\{f, g\}$ not to mention $\left\{f, \alpha_{t} g\right\}$ will not be bounded (or even necessarily exist) even if $f$ and $g$ are very nice. This introduces various technical problems. The appropriate (mostly technical) assumptions on the time evolution necessary for our analysis of stability are given in Section 2 where we define a "regular time evolution". In Section 3 we prove the main result: stability plus proper clustering of the dynamical system $\left(K, \alpha_{t}, \omega\right)$ implies the $\mathrm{KMS}_{\mathrm{D}}$ condition.

The results, however, are not very satisfactory because of the large number of assumptions that have to be made. These are far from being provable with our present knowledge of the theory of the Hamiltonian equations for systems with infinitely many degrees of freedom. Indeed the only case for which the assumptions can be verified is the ideal gas. This is done in Section 4. Some remarks about the reasonableness of our assumptions and alternative formulations are made in Section 5 . The relation between stability and Gibbs states for the ideal gas can also be proven directly without the use of the KMS condition [17].

\section{Classical Systems}

A classical system of infinitely many particles moving in $R^{d}$ is described by the following three objects $[1,2]$ :

1) The "phase space", denoted by $K$, which consists of the sequences $X=\left(q_{i}, p_{i}\right)_{i=1}^{\infty} \equiv\left(x_{i}\right)_{1}^{\infty}$ of points $x=(q, p) \in R^{d} \times R^{d}$ with the "local finiteness" property: i.e. the number of points of $\bigcup_{i=1}^{\infty}\left\{q_{i}\right\}$ inside any bounded region is finite. Two sequences which differ only by a permutation are regarded as identical.

2) The "algebra of the unbounded strictly local observables", denoted $\tilde{\mathfrak{A}}$, which consists of the functions $f: K \rightarrow R$ which can be described in terms of a sequence $\left(f^{(m)}\right)_{0}^{\infty}$ of $C^{\infty}$-functions $f^{(m)}:\left(R^{d} \times R^{d}\right)^{m} \rightarrow R$ which are symmetric, have 
compact support and are identically zero for all but finitely many values of $m$. The expression for $f$ in terms of $\left(f^{(m)}\right)_{0}^{\infty}$ is given by

$$
f(X)=\sum_{m=0}^{\infty} \sum_{i_{1}<\ldots<i_{m}} f^{(m)}\left(x_{i_{1}}, \ldots, x_{i_{m}}\right) \equiv \sum_{m=0}^{\infty}\left(\sum f^{(m)}\right) .
$$

The space $K$ will be regarded as a topological space with the smallest topology which makes all $f \in \tilde{\mathfrak{U}}$ continuous. For technical reasons we shall be working primarily not with $\tilde{\mathfrak{U}}$ but with $\mathfrak{U}$ : the algebra of bounded strictly local observables generated by the functions $G: K \rightarrow R$ of the form

$$
G(X)=\Psi(f(X))
$$

with $f \in \tilde{\mathfrak{U}}$ and $\Psi: R \rightarrow R$ a $C^{\infty}$-function bounded together with its derivatives.

3) A "state", denoted by $\omega$, is a Lebesgue probability measure on the Borel $\sigma$-field of $K$ such that $\tilde{\mathfrak{A}}$ is densely contained in $L^{2}(\omega)$.

We introduce a "Poisson bracket" via the following definition:

Definition 1. Let $f, g \in \mathfrak{U}$, define $\{f, g\}=\sum_{i \equiv 1}^{\infty}\left(\frac{\partial f}{\partial q_{i}} \frac{\partial g}{\partial p_{i}}-\frac{\partial f}{\partial p_{i}} \frac{\partial g}{\partial q_{i}}\right)$ where $\frac{\partial}{\partial q_{i}} \frac{\partial}{\partial p_{i}}$ denotes the scalar of the gradients. The map $\{.,$.$\} is a bilinear map from$ $\mathfrak{U} \times \mathfrak{U} \rightarrow C(K)=$ continuous functions on $K$. It will be called the Poisson bracket $(\mathrm{PB})$.

\section{Time Evolution of Classical Systems}

The time evolution of a classical system should be described by the following chain of definitions.

Definition 2. Let $\omega$ be a state of a classical system. Suppose that there is an $\omega$-measurable full set $\mathscr{H}_{\omega} \subset K$ on which a group $\alpha$ of maps $\alpha_{t}: \mathscr{H}_{\omega} \rightarrow \mathscr{H}_{\omega}, t \in \mathbb{R}$, is defined such that;

a) if $\left(q_{i}(t), p_{i}(t)\right)_{i=1}^{\infty}=\alpha_{t}\left(q_{i}, p_{i}\right)_{i=1}^{\infty}$ then $q_{i}(t), p_{i}(t)$ are differentiable functions of $t$,

b) the operators

$$
(U(t) f)(X)=f\left(\alpha_{t} X\right)
$$

define a strongly continuous group of operators on $L^{2}(\omega)$ whose generator $\mathscr{L}$ has a domain which contains $\mathfrak{U}$

c) if $\left(q_{i}, p_{i}\right)_{1}^{\infty} \in \mathscr{H}_{\omega}$ and if $\left(\dot{q}_{i}, \dot{p}_{i}\right)_{1}^{\infty}=\left.\left(\dot{q}_{i}(t), \dot{p}_{i}(t)\right)_{1}^{\infty}\right|_{t=0}=\left(\Phi_{i}^{1}, \Phi_{i}^{2}\right)_{1}^{\infty}$, then $\forall f \in \mathfrak{A}$

$$
\mathscr{L} f=\sum_{i=1}^{\infty}\left(\dot{q}_{i} \frac{\partial f}{\partial q_{i}}+\dot{p}_{i} \frac{\partial f}{\partial p_{i}}\right)=\sum_{i=1}^{\infty}\left(\Phi_{i}^{1} \frac{\partial f}{\partial q_{i}}+\Phi_{i}^{2} \frac{\partial f}{\partial p_{i}}\right)
$$

everywhere on $\mathscr{H}_{\omega}$. When this holds then $\alpha$ is called a time evolution for $\omega$.

$\omega$ is a stationary state for $\alpha$ if $U(t)$ is a unitary operator for all $t$. (We shall generally write $\alpha_{t} f$ for $U(t) f$.)

Remark. If $\omega$ is a Gibbs state for a Hamiltonian $H$ with pair-potential $\varphi$ the time evolution associated to the Hamiltonian equations:

$$
\Phi_{i}^{1}=p_{i} / m, \Phi_{i}^{2}=-\sum_{j \neq i} \frac{\partial \varphi\left(q_{i}-q_{j}\right)}{\partial q_{i}}
$$

is a time evolution $[2,7,9]$ having all the above properties, and leaves $\omega$ invariant. 
Definition 3. Let $\omega$ be a state of a classical system and let $\alpha$ be a time evolution for it. We shall say that $\alpha$ is a regular time evolution for $\omega$ when the following properties hold.

i) Let $\mathfrak{A}(\omega, \alpha)=\bigvee_{t \in R} \alpha_{t} \mathfrak{A}\left(=\right.$ algebra generated by the functions in $\alpha_{t} \mathfrak{A}$, $\left.\mathrm{t} \in R\right)$. The series ${ }^{1}$

$$
\{f, g\}=\sum_{i=1}^{\infty}\left[\frac{\partial f}{\partial q_{i}} \frac{\partial g}{\partial p_{i}}-\frac{\partial f}{\partial q_{i}} \frac{\partial g}{\partial p_{i}}\right]
$$

makes sense term by term and is absolutely convergent in $\mathscr{H}_{\omega}$ and $\{f, g\} \in L^{2}(\omega)$, $\forall f, g \in \mathfrak{U}(\omega, \alpha)$.

ii) If $h \in \mathfrak{U}(\omega, \alpha)$ the equations

$$
\begin{aligned}
& \dot{q}_{i}=\Phi_{i}^{1}+\frac{\partial h}{\partial p_{i}} \\
& \dot{p}_{i}=\Phi_{i}^{2}-\frac{\partial h}{\partial q_{i}}
\end{aligned}
$$

have a solution $\left(q_{i}(t), p_{i}(t)\right)_{1}^{\infty}=\alpha_{t}^{h}\left(q_{i}, p_{i}\right)_{i=1}^{\infty} \in \mathscr{H}_{\omega}$ for all $\left(q_{i}, p_{i}\right)_{1}^{\infty} \in \mathscr{H}_{\omega}$ such that $\alpha^{h}$ verifies the properties a)-c) of a time evolution for $\omega$ with $\mathfrak{A}(\omega, \alpha)$ replacing $\mathfrak{A}$ (see Def. 2).

The generator $\mathscr{L}^{h}$ of the strongly continuous group of operators $U^{h}(t)$ on $L^{2}(\omega)$ is, by our assumptions,

$$
\mathscr{L}^{h} f=\mathscr{L} f+\{f, h\} \quad \forall f \in \mathfrak{A}(\omega, \alpha) .
$$

Remark. There is no interacting classical system for which it is known that the time evolution is regular.

Let us denote by $\mathscr{B}_{\Lambda}$, for $\Lambda \subset \mathbb{R}^{d} \times \mathbb{R}^{d}$, the collection of $\omega$-measurable functions $f$ on $K$ such that $f(X)$ depends only on the points $x_{i} \in \Lambda$.

Definition 4. A collection of functions $\mathscr{D}$ is locally dense in $L^{2}(\omega)$ if $\mathscr{D} \cap \mathscr{B}_{A}$ is dense, in the $L^{2}(\omega)$ norm, in $L^{2}(\omega) \cap \mathscr{B}_{\Lambda}$ for any closed $\Lambda \subset \mathbb{R}^{d} \times \mathbb{R}^{d}$.

\section{Main Theorem}

Let $Y=(K, \alpha, \omega)$ be a classical dynamical system with a regular time evolution generated by a Hamiltonian $H$. Let $\mathscr{D}$ be a locally dense subalgebra of $\mathfrak{A}(\omega, \alpha)$ which is invariant under $\alpha$. We make the following further definitions:

5) $Y$ is dispersive if

$$
\left\|\left\{f, \alpha_{t} g\right\}\right\|_{L^{2}(\omega)} \in L^{1}(\mathbb{R}) \text { as a function of } t, \quad \forall f, g \in \mathscr{D} \text {. }
$$

6) $Y$ is 3-fold mixing if $\forall$ bounded $f_{i}, i=1,2,3$

$$
\omega\left(\alpha_{t_{1}} f_{1} \alpha_{t_{2}} f_{2} \alpha_{t_{3}} f_{3}\right) \underset{\substack{\min \left|t_{i}-t_{j}\right| \rightarrow \infty \\ i \neq j}}{\longrightarrow} \omega\left(f_{1}\right) \omega\left(f_{2}\right) \omega\left(f_{3}\right) .
$$

1 Notice that in order to be able to define $\{.,$.$\} on \mathscr{H}_{\omega}$ one needs to assume that $\mathscr{H}_{\omega}$ is locally stable: i.e. if $X=\left(q_{i}, p_{t}\right)_{1}^{\infty} \in \mathscr{H}_{\omega}$ and $\left(\delta_{i}\right)_{1}^{\infty}$ is a sequence of vectors in $\mathbb{R}^{d} \times \mathbb{R}^{d}$ which is identically zero for all but one index then $X^{\prime}=\left(x_{i}+\delta_{i}\right)_{1}^{\infty} \in \mathscr{H}_{\omega}$. 
7) $Y$ is stable under the perturbation $h \in \mathscr{D}$ if for some $\delta>0$ and for each $\lambda \in[0, \delta) \exists$ a state $\omega^{\lambda h}$ which is

i) invariant under the time evolution $\alpha_{t}^{\lambda h}$,

ii) absolutely continuous with respect to $\omega$ and given by an $L^{2}(\omega)$ density function

iii) $\omega^{\lambda h}$ converges weakly to $\omega$ on $L^{2}(\omega)$, i.e.

$\lim _{\lambda \rightarrow 0} \omega^{\lambda h}(g)=\omega(g), \quad \forall g \in L^{2}(\omega)$.

8) $Y$ is stable if it is stable under the perturbation $h$, for all $h \in \mathscr{D}$.

Remark. The assumption that $\mathscr{D}$ is locally dense in $\mathfrak{A}(\omega, \alpha)$ will only be used in [16] where it is needed for proving that the $\mathrm{KMS}_{\mathrm{E}}$ condition implies that the state is a Gibbs state.

Our main result is the following theorem:

Theorem 1. Let $\omega$ be an invariant state under a regular time evolution $\alpha_{t}$ which is dispersive and 3-fold mixing. If $\omega$ is stable then it is a $\mathrm{KMS}_{\mathrm{D}}$ state, i.e. it satisfies (1.3).

We shall first prove the following lemma.

Lemma 1. Under the assumptions of the theorem, $\forall f_{i}, g_{i} \in \mathscr{D}$ :

i) $\int_{-\infty}^{\infty} G_{i}(t) d t=0$

ii) $\int_{-\infty}^{\infty} \sum_{k=1}^{N} F_{1}(t) F_{2}(t) \ldots G_{k}(t) \ldots F_{N}(t) d t=0, \forall N \in \mathbb{Z}_{+}$,

where $F_{i}(t)$ and $G_{i}(t)$ stand respectively for $F_{f_{l}, g_{1}}(t)$ and $G_{f_{l}, g_{l}}(t)$ defined in $(1.1)$ and (1.2).

Notice that all the above integrands are absolutely intergrable since the $F_{i}(t)$ are bounded and, by virtue of the dispersive property, $G_{i}(t) \in L^{1}(\mathbb{R}) \forall f_{i}, g_{i} \in \mathscr{D}$. We also note that i) and ii) are satisfied in any mixing $\mathrm{KMS}_{\mathrm{D}}$ state.

Proof. Let $f$ and $g$ belong to $\mathscr{D}$. The stability of $\omega$ under $f$ as a perturbation implies the existence of states $\omega^{\lambda f}$ (for $\delta>\lambda \geqq 0$ ) such that $\mathscr{D} \subset L^{1}\left(\omega^{\lambda f}\right)$ and which are invariant under the perturbed time evolution. It follows from the latter that

$$
\omega^{\lambda f}\left(\mathscr{L}^{\lambda f} \alpha_{t} g\right)=0
$$

which, by (2.3), implies that

$$
\omega^{\lambda f}\left(\left\{f, \alpha_{t} g\right\}\right)=\frac{1}{\lambda} \omega^{\lambda f}\left(\mathscr{L} \alpha_{t} g\right)=\frac{1}{\lambda} \frac{d}{d t} \omega^{\lambda f}\left(\alpha_{t} g\right) .
$$

Integrating both sides of this equation from $-\tau$ to $\tau$ and taking the limit $\tau \rightarrow \infty$ we obtain

$$
\begin{aligned}
\int_{-\infty}^{\infty} \omega^{\lambda f}\left(\left\{f, \alpha_{t} g\right\}\right) d t & =\lambda^{-1} \int_{-\infty}^{\infty} \frac{d}{d t} \omega^{\lambda f}\left(\alpha_{t} g\right) d t \\
& =\lim _{\tau \rightarrow \infty} \lambda^{-1}\left[\omega^{\lambda f}\left(\alpha_{\tau} g\right)-\omega^{\lambda f}\left(\alpha_{-\tau} g\right)\right]=0,
\end{aligned}
$$

where the last equality follows from mixing. As $\lambda \rightarrow 0 \omega^{\lambda f}$ converges weakly to $\omega$ (on $L^{2}(\omega)$ ) which implies (by the principle of uniform boundedness) that the $L^{2}(\omega)$ 
norms of the densities which give $\omega^{\lambda f}$ are bounded uniformly in $\lambda$, and thus

$$
\omega^{\lambda f}\left(\left\{f, \alpha_{t} g\right\}\right) \leqq C\left\|\left\{f, \alpha_{t} g\right\}\right\|_{L^{2}(\omega)} \in L^{1}(\mathbb{R}) .
$$

Hence one may interchange the limit $\lambda \rightarrow 0$ with the integration in (3.1) (using the dominated convergence theorem) to obtain i).

The condition on $\omega$ expressed in i) makes no reference to the existence of approximating states $\omega^{\lambda f}$ and, as it turns out, the derivation of i) is the only place in the proof where the stability of $\omega$ is used directly.

ii) Will be proven by induction on $N$ starting from i) $(N=1)$. As an induction step, let us assume ii) to hold for some $N \geqq 1$ and any $f_{i}, g_{i} \in \mathscr{D}, i=1, \ldots, N$ and prove it for $N+1$ and a given $f_{i}, g_{i} \in \mathscr{D}, i=1, \ldots, N+1$. We denote, for some $u \in \mathbb{R}$,

$$
\begin{aligned}
& \tilde{f}=f_{N} \alpha_{u} f_{N+1}, \quad \tilde{g}=g_{N} \alpha_{u} g_{N+1} \\
& \tilde{F}(t)=F_{\tilde{f}, \tilde{g}}(t) \quad \text { and } \quad \tilde{G}(t)=G_{\tilde{f}, \tilde{g}}(t) .
\end{aligned}
$$

The induction assumption, when applied to $\left\{F_{1}, \ldots, F_{N-1}, \tilde{F}\right\}$, implies

$$
\int \sum_{k=1}^{N-1} F_{1}(t) \ldots G_{k}(t) \ldots F_{N-1}(t) \tilde{F}(t) d t+\int F_{1}(t) \ldots F_{N-1}(t) \tilde{G}(t) d t=0 .
$$

We shall now take the limit $u \rightarrow \infty$ using the 3-fold mixing and dispersiveness of $\omega$.

First, notice, using well known properties of the PB and the mixing property of the unperturbed time evolution, that

$$
\begin{aligned}
\tilde{G}(t)= & \omega\left(f_{N} \cdot \alpha_{t} g_{N} \cdot \alpha_{u}\left\{f_{N+1}, \alpha_{t} g_{N+1}\right\}\right)+\omega\left(\left\{f_{N}, \alpha_{t} g_{N}\right\} \alpha_{u}\left(f_{N+1} \alpha_{t} g_{N+1}\right)\right) \\
& +\omega\left(f_{N} \cdot \alpha_{t+u} g_{N+1} \cdot\left\{\alpha_{u} f_{N+1}, \alpha_{t} g_{N}\right\}\right) \\
& +\omega\left(\alpha_{u} f_{N+1} \cdot \alpha_{t} g_{N} \cdot\left\{f_{N}, \alpha_{t+u} g_{N+1}\right\}\right) \\
= & \tilde{G}_{1}+\tilde{G}_{2}+\tilde{G}_{3}+\tilde{G}_{4}
\end{aligned}
$$

where

$$
\begin{aligned}
& \tilde{G}_{1}(t) \underset{u \rightarrow \infty}{\longrightarrow} F_{N}(t) G_{N+1}(t)+\omega\left(f_{N}\right) \omega\left(g_{N}\right) G_{N+1}(t) \\
& \tilde{G}_{2}(t) \underset{u \rightarrow \infty}{\longrightarrow} G_{N}(t) F_{N+1}(t)+G_{N}(t) \omega\left(f_{N+1}\right) \omega\left(g_{N+1}\right) .
\end{aligned}
$$

Also:

$$
\begin{aligned}
\tilde{F}(t)= & \omega\left(\left(f_{N} \alpha_{t} g_{N}\right) \alpha_{u}\left(f_{N+1} \alpha_{t} g_{N+1}\right)\right)-\omega\left(f_{N} \alpha_{u} f_{N+1}\right) \omega\left(g_{N} \alpha_{u} g_{N+1}\right) \\
& \underset{u \rightarrow \infty}{\longrightarrow} F_{N}(t) F_{N+1}(t)+F_{N}(t) \omega\left(f_{N+1}\right) \omega\left(g_{N+1}\right)+\omega\left(f_{N}\right) \omega\left(g_{N}\right) F_{N+1}(t) .
\end{aligned}
$$

The dispersive property of $\omega$ guarantees that the terms of (3.3) which involve $\tilde{F}(t)$, $\tilde{G}_{1}(t)$, and $\tilde{G}_{2}(t)$ are bounded by an $L^{1}(\mathbb{R})$ function of $t$, independent of $u$. Hence in these terms one may interchange the limit $u \rightarrow \infty$ with the intergration and, applying the induction assumption to $\left\{F_{1}, \ldots, F_{N-1}, F_{N}\right\}$ and to $\left\{F_{1}, \ldots, F_{N-1}, F_{N+1}\right\}$, it is easy to see that they converge to

$$
\int \sum_{k=1}^{N+1} F_{1}(t) \ldots G_{k}(t) \ldots F_{N+1}(t) d t .
$$


As to the remaining terms, those may be rearranged as follows:

$$
\begin{aligned}
\int F_{1}(t) \ldots F_{N-1}(t)\left[\tilde{G}_{3}(t)+\tilde{G}_{4}(t)\right] d t & \\
= & \omega\left(f_{N}\right) \omega\left(g_{N+1}\right) \int F_{1}(t) \ldots F_{N-1}(t) \omega\left(\left\{\alpha_{u} f_{N+1}, \alpha_{t} g_{N}\right\}\right) d t \\
+ & \omega\left(g_{N}\right) \omega\left(f_{N+1}\right) \int F_{1}(t) \ldots F_{N-1}(t) \omega\left(\left\{f_{N}, \alpha_{t} \alpha_{u} g_{N+1}\right\}\right) d t \\
+ & \int F_{1}(t+u) \ldots F_{N-1}(t+u)\left[\omega\left(\alpha_{-u} f_{N} \cdot \alpha_{t+u} g_{N+1} \cdot\left\{f_{N+1}, \alpha_{t} g_{N}\right\}\right)\right. \\
& \left.\quad \omega\left(f_{N}\right) \omega\left(g_{N+1}\right) \omega\left(\left\{f_{N+1}, \alpha_{t} g_{N}\right\}\right)\right] d t \\
+\int F_{1}(t-u) \ldots F_{N-1}(t-u)\left[\omega\left(\alpha_{u} f_{N+1} \cdot \alpha_{t-u} g_{N} \cdot\left\{f_{N}, \alpha_{t} g_{N+1}\right\}\right)\right. & \left.\quad \omega\left(f_{N+1}\right) \omega\left(g_{N}\right) \omega\left(\left\{f_{N}, \alpha_{t} g_{N+1}\right\}\right)\right] d t .
\end{aligned}
$$

As $u \rightarrow \infty$ the last two integrals converge to zero since their integrands do so pointwise (3-fold mixing) while remaining bounded, uniformly in $u$, by

$$
C_{1}\left\|\left\{f_{N+1}, \alpha_{t} g_{N}\right\}\right\|_{L^{2}(\omega)} \in L^{1}(\mathbb{R}) \quad \text { and by } \quad C_{2}\left\|\left\{f_{N}, \alpha_{t} g_{N+1}\right\}\right\|_{L^{2}(\omega)} \in L^{1}(\mathbb{R}),
$$

respectively. The remaining terms, after applying again the induction assumption, are

$$
\begin{aligned}
& -\omega\left(f_{N}\right) \omega\left(g_{N+1}\right) \int \sum_{k=1}^{N-1} F_{1}(t) \ldots G_{k}(t) \ldots F_{N-1}(t) \omega^{T}\left(f_{N+1}, \alpha_{t-u} g_{N}\right) d t \\
& -\omega\left(g_{N}\right) \omega\left(f_{N+1}\right) \int \sum_{k=1}^{N-1} F_{1}(t) \ldots G_{k}(t) \ldots F_{N-1}(t) \omega^{T}\left(f_{N}, \alpha_{t+u} g_{N+1}\right) d t \\
& \underset{u \rightarrow \infty}{\longrightarrow} 0 \text { (bounded convergence th. }) .
\end{aligned}
$$

We thus obtain ii) for $N+1$, which by induction concludes the proof of the lemma.

To prove Theorem 1 using Lemma 1 we will use the following result whose proof is delayed to Appendix II.

Lemma 2. If $0 \neq F \in C_{\infty}^{1}(\mathbb{R})$ (i.e. $F \in C^{1}(\mathbb{R})$ and $F(t) \rightarrow 0$ as $|t| \rightarrow \infty$ ) and $G \in L^{1}(\mathbb{R})$ satisfy

$$
\int \sum_{k=1}^{N} F\left(t+\tau_{1}\right) F\left(t+\tau_{2}\right) \ldots G\left(t+\tau_{k}\right) \ldots F\left(t+\tau_{N}\right) d t=0
$$

$\forall N \in \mathbb{Z}_{+}$and $\left\{\tau_{1}, \ldots, \tau_{N}\right\} \subset \mathbb{R}^{N}$, then

$$
G(t)=-\beta \frac{d}{d t} F(t) \text { (at almost all } t \in \mathbb{R} \text { ) for some } \beta \in \mathbb{R} \text {. }
$$

Proof of Theorem 1. Let $f, g \in \mathscr{D}$ and consider, for some $\left\{\tau_{1}, \ldots, \tau_{N}\right\} \subset \mathbb{R}$, the $F$ and $G$ functions which correspond to

$$
f_{i}=f, g_{i}=\alpha_{\tau_{l}} g \text {. }
$$

Clearly

$$
F_{i}(t)=\omega^{T}\left(f, \alpha_{t+\tau_{\imath}} g\right)=F\left(t+\tau_{i}\right)
$$

and

$$
G_{i}(t)=\omega\left(\left\{f, \alpha_{t+\tau_{i}} g\right\}\right)=G\left(t+\tau_{i}\right) .
$$


Thus, by Lemma 1 (where our assumptions are needed):

$$
\int \sum_{k=1}^{N} F\left(t+\tau_{1}\right) F\left(t+\tau_{2}\right) \ldots G\left(t+\tau_{k}\right) \ldots F\left(t+\tau_{N}\right) d t=0 .
$$

Since the given $F$ and $G$ functions satisfy the above equation for any $N \in \mathbb{Z}_{+}$and $\left\{\tau_{1}, \ldots, \tau_{N}\right\} \in \mathbb{R}$, it follows by Lemma 2 that

$$
\left.G(t)=-\beta \frac{d}{d t} F(t) \quad \text { (for almost all } t \in \mathbb{R}\right) \text { with some } \beta \in \mathbb{R} .
$$

The above argument was carried out for given $f, g \in \mathscr{D}$ assuming $F \neq 0$. The fact that (3.4) is valid generally and that the constant $\beta$ is independent of the particular pair of observables follows from the fact that $F$ and $G$ are bilinear in $f$ and $g$.

Thus we have shown that $\omega$ is a $\mathrm{KMS}_{\mathrm{D}}$ state with some $\beta$ which, for consistency (see $[15,16])$, has to be positive.

\section{Stable States of the Ideal Gas System}

It is interesting to apply the criterion of stability to states of the infinite ideal gas system. As mentioned in the introduction this system has many stationary states which are not Gibbs equilibrium states although they satisfy all the usual regularity conditions.

The special features of the ideal gas enable one to state more explicitly for what states the assumptions (which were used in conjunction with stability) in Theorem 1 will be satisfied.

Let us denote by $\Gamma=\mathbb{R}^{d} \otimes \mathbb{R}^{d}$ the one particle phase space and by $\alpha_{t}^{(1)}$ the free time evolution on it:

$$
\alpha_{t}^{(1)}(q, p)=(q+t p / m, p) .
$$

The free time evolution of the ideal gas, under which the particles move independently, is generated by the formal Hamiltonian $H=\sum p_{i}^{2} / 2 m$ and may conveniently be described as

$$
\alpha_{t}\left\{\left(q_{i}, p_{i}\right)\right\}_{i=1}^{\infty}=\left\{\alpha_{t}^{(1)}\left(q_{i}, p_{i}\right)\right\}_{i=1}^{\infty} .
$$

Thus we also have for $f \in \tilde{\mathfrak{A}}$, Equation (2.1),

$$
\alpha_{t} f(X)=\sum_{n=0}^{\infty}\left\{\sum_{i_{1}<i_{2}<\ldots i_{n}} \alpha_{t}^{(n)} f^{(n)}\left(x_{i_{1}}, \ldots, x_{i_{n}}\right)\right\},
$$

where $\alpha_{t}^{(n)} f^{(n)}\left(x_{1}, \ldots, x_{n}\right)=f^{(n)}\left(\alpha_{t}^{(1)} x_{1}, \ldots, \alpha_{t}^{(1)} x_{n}\right)$. This feature is a consequence of the lack of interactions and it implies that for the ideal gas $\mathfrak{U}=\mathfrak{U}(\omega, \alpha)$. Thus the generator $\mathscr{L}$ of the time evolution, see Definition 2 , is the closure of its restriction $\sum \frac{p_{i}}{m} \frac{\partial}{\partial q_{i}}$ to $\mathfrak{A}$ (in other words $\mathscr{L}$ is essentially anti-selfadjoint on $\mathfrak{A}$, c.f. Ref. [15b]).

Let $f \in \tilde{\mathfrak{A}}$, we define $\Lambda_{f} \in \Gamma$ to be the smallest set such that

$$
\operatorname{supp}\left(f^{(n)}\right) \subset A_{f}^{n} \subset \Gamma^{(n)}, \quad \forall n \in Z_{+} .
$$


In order to apply the general result to the ideal gas system we define $\mathscr{D} \subset \mathfrak{U}$ as the sub-algebra generated by functions $\Psi(f)$ with $f \in \tilde{\mathfrak{U}}$ such that $\Lambda_{f}^{n}$ excludes the subspaces $\left\{p_{i}=0\right\} i=1, \ldots, n$. It is easy to see that $\mathscr{D}$ is locally dense with respect to any state which locally is absolutely continuous with respect to the Liouville measure. Let $f, g \in \mathscr{D}$, since $\Lambda_{f}$ and $\alpha_{t}^{(1)} \Lambda_{g}$ separate after a finite time $T$, we obtain

$$
\left\{f, \alpha_{t} g\right\} \equiv 0 \text { for }|t|>T
$$

which implies that any state of the ideal gas for which $\mathscr{D}$ is locally dense is dispersive. The three fold mixing property will also be possessed by many stationary states of the ideal gas, e.g. if the correlation functions are products of the one particle correlation function since such states are Bernoulli [10].

It is interesting to notice that the above restriction on $\mathscr{D}$ is necessary: for a general $f \in \mathfrak{A}\left\|\left\{f, \alpha_{t} f\right\}\right\|_{L^{2}(\omega)}$ is not integrable as a function of time. In fact the leading term in $\left\{f, \alpha_{t} f\right\}$, for large $t$ and $f=\sum f^{(1)}$, is

$$
t / m \sum \frac{\partial f^{(1)}}{\partial q_{i}} \alpha_{t} \frac{\partial f^{(1)}}{\partial q_{i}}
$$

The increasing factor $t$ reflects the instability of the time evolution: a small change in the initial configuration, $\left(q_{i}, p_{i}\right) \rightarrow\left(q_{i}, p_{i}+\lambda \delta_{i, j}\right)$, results in a growing deviation of the configurations at time $t$. However, the summands in (4.2) are non zero only if $q_{i} \in \Lambda_{f} \cap \alpha_{-t}^{(1)} \Lambda_{f}$ which restricts $q_{i}$ to a volume of the order of $C / t$ in $\Gamma$ (that is, in case $\Lambda_{f}$ is not bounded away from $\{p=0\}$ ). Hence the $L^{2}(\omega)$ norm of (4.2) need not even decay to zero.

\section{Remarks on the Dispersive Property and Other Matters}

1. One may expect the instability of the time evolution, which leads to the increasing factor in (4.1) to be generic. It is easy to convince oneself that for finite systems $\left\|\left\{f, \alpha_{t} f\right\}\right\|_{L^{2}(\omega)} \rightarrow 0$ as $t \rightarrow \infty$, and that for an infinite system the decay of the above norm is a measure of some sort of dispersion (hence the term). At this point we lack any general result about the existence of such dispersion in systems of interacting particles.

2. One may interpret the dispersive property in a way which relates the proof of Theorem 1 to the method used for finite system. A consequence of stability, which is useful when dealing with the finite classical and quantum system [6], is the invariance of the state under any flow whose generator is an admissible perturbation invariant under $\alpha_{t}$. Therefore stability is a strong restriction on the state whenever the system has a large class of "generators" which commute with the Hamiltonian.

We note now that, for a given $f \in \mathscr{D}$, the condition

$$
\left\|\left\{\alpha_{t} f, g\right\}\right\|_{L^{2}(\omega)} \in L^{1} \quad \forall g \in \mathscr{D} \subset L^{2}(\omega)
$$

is exactly what is necessary in order to be able to define an operator $\mathscr{M}_{f}$ on the domain $\mathscr{D}$ by the Bochner integral:

$$
\mathscr{M}_{f} \cdot g=\int_{-\infty}^{\infty} d t\left\{\alpha_{t} f, g\right\}
$$


The operators $\mathscr{M}_{f}$ defined in this way act as derivations on $\mathscr{D}$ and commute with the time evolution:

$$
\begin{aligned}
\mathscr{M}_{f}\left(\alpha_{\tau} g\right) & =\int_{-\infty}^{\infty} d t\left\{\alpha_{t} f, \alpha_{\tau} g\right\} \\
& =\alpha_{\tau} \int_{-\infty}^{\infty} d t\left\{\alpha_{t} f, g\right\}=\alpha_{\tau} \mathscr{M}_{f} g .
\end{aligned}
$$

Hence the dispersive property guarantees the existence of a "large" class of "pseudo-generators" defined on a "large" domain, which commute with the time evolution. It is thus not surprising that a condition of this type was needed in Theorem 1.

Part i) of Lemma 1, which was the only direct consequence of stability used in the proof of Theorem 1, may now be rewritten as:

$$
\omega\left(\mathscr{M}_{f} \cdot g\right)=0 \quad \forall f, g \in \mathscr{D},
$$

which looks like an invariance property of the type encountered for finite systems. [In view of the derivation property of $\mathscr{M}_{f}$ it implies that $\mathscr{M}_{f}$ is anti-hermitian in $L^{2}(\omega)$.]

3. As already mentioned in the introduction and as is clear now, the fact that $\mathscr{D}$ is locally dense in $\mathfrak{U}(\omega, \alpha)$ has not been used at all in the proof of $\mathrm{KMS}_{\mathrm{D}}$ condition. It will however be needed in the proof [16] that $\mathrm{KMS}_{\mathrm{E}}$ implies that $\omega$ is a "canonical" equilibrium state with temperature $\beta^{-1}$. The precise definition of $\mathfrak{U}(\omega, \alpha)$ is however far from unique: $\mathfrak{A}$ could be replaced by other large enough algebras $\mathfrak{A}^{\prime}$ of functions which are such that the series defining the Poisson brackets $\{f, g\}, f, g \in \mathfrak{H}^{\prime}$, are convergent [17].

4. When dealing with finite classical systems in Ref. 6 we introduced the notion of "dynamical stability": if the system is in a state $\omega$ stationary under the time evolution generated by the Hamiltonian $H$ then if $H$ is changed, say at $t=0$, by a small perturbation $\lambda h$ then this should cause only a small change in the expectation values of the observables $f$ for all times. This appears to us to be a physically very reasonable requirement on an equilibrium state and is expressed formally as

$$
\left|\omega\left(\alpha_{t}^{\lambda h} f\right)-\omega(f)\right| \leqq \varepsilon(\lambda) \sup _{X}|f|
$$

with $\varepsilon(\lambda) \rightarrow 0$ as $\lambda \rightarrow 0$.

We noted there and this is true also here that if the HKT-P stability, Definition 7, iii) is changed to require that $\left|\omega^{\lambda h}(f)-\omega(f)\right| \leqq \varepsilon(\lambda) \sup _{X}|f|$ then it implies (5.3) since the existence of an $\omega^{\lambda h}$ with the property that $\omega^{\lambda h}\left(\alpha_{t}^{\lambda h} f\right)=\omega^{\lambda h}(f)$ implies that

$$
\left|\omega\left(\alpha_{t}^{\lambda h} f\right)-\omega(f)\right| \leqq\left|\omega\left(\alpha_{t}^{\lambda h} f\right)-\omega^{\lambda h}\left(\alpha_{t}^{\lambda h} f\right)\right|+\left|\omega^{\lambda h}(f)-\omega(f)\right| .
$$

The converse statement is also true, at least in a weak sense, since we can then construct $\omega^{\lambda h}(f)$ by taking the Cesaro mean of $\omega\left(\alpha_{t}^{\lambda h} f\right)$ for a suitable large class of functions $f$. 
5. The dispersion assumption is, as already noted, the most questionable one for general interacting systems. It is used many times in the course of the proof of Theorem 1 to insure the convergence of various integrals such as the one occuring in (3.1). We merely wish to note here that one could carry through the proof, albeit more clumsily, by "merely" assuming in each place where necessary, that the integrals converge and that limits can be interchanged.

Acknowledgements. We would like to thank Dr. Rudolph Haag and Dr. Eva Trych-Pohlmeyer for informing and explaining to us their results prior to publication and for valuable discussions, and to Dr. Henry McKean for a valuable discussion regarding Lemma 2.

\section{Appendix A : Proof of Lemma 2}

Lemma. If $0 \neq F \in C_{\infty}^{1}(\mathbb{R})$ (i.e. $F \in C^{1}(\mathbb{R})$ and $F(t) \underset{|t| \rightarrow \infty}{\longrightarrow} 0$ ) and $G \in L^{1}(\mathbb{R})$ satisfy

$$
\begin{aligned}
& \int \sum_{k=1}^{N} F\left(t+\tau_{1}\right) F\left(t+\tau_{2}\right) \ldots G\left(t+\tau_{k}\right) \ldots F\left(t+\tau_{N}\right) d t=0, \quad d t=0, \forall N \in \mathbb{Z}_{+} \\
& \text {and } \forall\left\{\tau_{1}, \ldots, \tau_{N}\right\} \in \mathbb{R}^{N},
\end{aligned}
$$

then

$$
G(t)=\beta \frac{d}{d t} F(t) \quad(\text { for almost all } t \in \mathbb{R})
$$

for some $\beta \in \mathbb{R}$.

Proof. Let $N \in \mathbb{Z}_{+}$and $\left\{\tau_{1}, \ldots, \tau_{N}\right\} \in \mathbb{R}^{N}$ and denote

$$
\underline{R}(t)=\left\{F\left(t+\tau_{1}\right), \ldots, F\left(t+\tau_{N}\right)\right\} \in \mathbb{R}^{N} \quad\left(\underline{R}(t)=\underline{R}_{N ; \tau_{1}, \ldots, \tau_{N}}(t)\right)
$$

and

$$
\underline{G}(t)=\left\{G\left(t+\tau_{1}\right), \ldots, G\left(t+\tau_{N}\right)\right\} \in \mathbb{R}^{N} \quad\left(\underline{G}(t)=\underline{G}_{N ; \tau_{1}, \ldots, \tau_{N}}(t)\right) .
$$

We consider $\underline{R}(t)$ as a curve in $\mathbb{R}^{N}$ which, as a consequence of $F$ being in $C_{\infty}^{1}(\mathbb{R})$, has the following properties:

i) It is contained in a compact region $[-B, B]^{N}$, where $0<B<\infty$ is a uniform bound on $F(t)$.

ii) $\underline{R}(t) \rightarrow 0$ as $|t| \rightarrow \infty$.

iii) $\underline{R}(t)$ is differentiable.

Let us first show that $\forall \Psi \in C^{1}\left(\mathbb{R}^{N}\right)$ :

$$
\int_{-\infty}^{\infty}(\underline{G}(t), \nabla \Psi(\underline{R}(t))) d t=0 .
$$

Since $G \in L^{1}(\mathbb{R})$ the above integral defines a continuous functional on $C^{1}\left([-B, B]^{N}\right)$ with the norm

$$
\|\Psi\|=\sup _{x \in[-B, B]^{N}}\{|\Psi(x)|+\|\underline{\Psi} \Psi(x)\|\} .
$$

To prove the claim observe that for monomials it reduces to (A.1) and thus holds true and extend, by linearity and continuity, to all $C^{1}\left([-B, B]^{N}\right)$ (in which the polynomials are dense with respect to the above norm). 
Now, let $S_{i} \in C^{1}(\mathbb{R})$ be a sequence of functions whose derivatives are uniformly bounded and converge pointwise to $\theta(x-a)$ (the Heaviside step function). By substituting $S_{i}(\Psi)$ in place of $\Psi$ in (2) we may conclude (using the bounded convergence theorem) that

$$
\begin{aligned}
& \forall N \in \mathbb{Z}, \quad \Psi \in C^{1}\left(\mathbb{R}^{N}\right) \text { and }-\infty \leqq a<\infty: \\
& I_{\Psi}(a)=\int_{\Psi(\underline{R}(t)) \geqq a}(\underline{G}(t), \underline{\nabla} \Psi(\underline{R}(t)) d t=0 .
\end{aligned}
$$

We shall use the above representation and the above result to show that $\forall \tau \in \mathbb{R}$ :

$$
\operatorname{det}\left(\begin{array}{ll}
F^{\prime}(t) & F^{\prime}(t+\tau) \\
G(t) & G(t+\tau)
\end{array}\right)=0
$$

for almost all $t \in \mathbb{R}$.

Let $\tau, t_{0} \in \mathbb{R}$. Since $\lim _{|t| \rightarrow \infty} F(t)=0$, one may find $2 \leqq N \in \mathbb{Z}_{+}$and a collection $\left\{\tau_{1}=0, \tau_{2}=\tau, \tau_{3}, \ldots, \tau_{N}\right\}$ for which

a) the coordinates of

$$
\underline{R}_{N ; \tau_{1}, \ldots, \tau_{N}}(t)=\left(F(t), F(t+\tau), F\left(t+\tau_{3}\right), \ldots, F\left(t+\tau_{N}\right)\right)
$$

define $t_{0}$ uniquely (in the following we shall omit the subscripts of $\underline{R}(t)$ )

b) $\underline{R}\left(t_{0}\right) \neq 0$,

c) $\left.\frac{d}{d t} \underline{R}(t)\right|_{t=t_{0}} \neq 0$.

We shall call $a \in \mathbb{R}$ a regular value of $\Psi \in C^{1}\left(\mathbb{R}^{N}\right)$ if

i) $a \neq \Psi(\underline{0})$

ii) $\Psi(\underline{R}(t))=a$ for at most a finite number of times: $t_{1}(a), \ldots, t_{n}(a)$

iii) $\left.\frac{d}{d t} \Psi(\underline{R}(t))\right|_{t=t_{i}(a)} \neq 0 \quad 1 \leqq i \leqq n$.

Let us now apply (A.3) to a function $\Psi \in C^{1}\left(R^{N}\right)$ for which $\Psi\left(\underline{R}\left(t_{0}\right)\right)=a_{0}$ is a regular value (the conditions $\mathrm{a})-\mathrm{c}$ ) guarantee that such functions exist). It is not difficult to see that the set of regular values is open and that the derivative of $I_{\Psi}(a)$ in some neighborhood $\eta$ of $a_{0}$, is given by

$$
0=\frac{d}{d t} I_{\Psi}(a)=\sum_{1 \leqq i \leqq n(a)} \frac{\left(\underline{G}\left(t_{i}(a)\right), \underline{\nabla} \Psi\left(\underline{R}\left(t_{i}\right)\right)\right)}{\left|\left(\underline{\dot{R}}\left(t_{i}(a)\right), \underline{\nabla} \Psi\left(\underline{R}\left(t_{i}\right)\right)\right)\right|}
$$

for almost any $a \in \eta$.

Equation (A.5) seems to relate the values of the gradient of $\Psi$ at a finite number of points of $\mathbb{R}^{N}$. However, any non trivial relation is excluded by the arbitrariness of $\Psi$. In particular, one may modify $\Psi$ in a small neighborhood of $\underline{R}\left(t_{0}\right)$ [see $(a)$ above]. (A.5) is therefore satisfied only if, for almost any $t$ in some neighborhood of $t_{0}$,

$$
\frac{(\underline{G}(t), \underline{V})}{(\underline{\dot{R}}(t), \underline{V})}=\beta(t)
$$


with some $\beta(t)$ independent of $\underline{V} \in R^{N}$. It follows that for almost any $t$, in the above neighborhood of $t_{0}, \underline{G}(t)$ and $\underline{\dot{R}}(t)$ are parallel and in particular (A.4) is satisfied. By the $\sigma$-compactness of $\mathbb{R}$ it follows that, for a given $\tau \in \mathbb{R},(\mathrm{A} .4)$ is satisfied for almost any $t \in \mathbb{R}$.

Thus, for a.a. $\left(t_{1}, t_{2}\right) \in \mathbb{R}^{2}$

$$
F^{\prime}\left(t_{1}\right) G\left(t_{2}\right)=F^{\prime}\left(t_{2}\right) G\left(t_{1}\right)
$$

which implies that for some $\beta \in \mathbb{R}$

$$
G(t)=\beta F^{\prime}(t) \text { at a.a. } t \in \mathbb{R} \text {, since } F \neq 0 .
$$

\section{References}

1. Ruelle, D.: Statistical mechanics. New York: Benjamin 1969

2. Lanford, O.: In: Dynamical systems, theory and applications. Lecture Notes in Physics, 38, (ed. J. Moser) Berlin-Heidelberg-New York: Springer 1975

3. Ruelle, D.: Commun. math. Phys. 18, 127 (1970)

4. Gruber, C., Lebowitz, J.L.: Commun. math. Phys. 41, 11 (1975)

5. Lanford, O.: In: Statistical mechanics and mathematical problems. Lecture Notes in Physics, 20, ed. A. Lenard. Berlin-Heidelberg-New York: Springer 1973;

Thompson, R. L.: Mem. Am. Math. Soc. 150 (1974);

Logan, K. G.: Thesis, Cornell Univ. (1974);

Giorgii,H.O.: Z. Wahrscheinlichkeitstheorie verw. Geb. 32, 277 (1975): On Canonical Gibbs States and Symmetric and Tail Events (preprint) see also Ref. 16

6. Lebowitz, J. L., Aizenman, M., Goldstein, S.: J. Math. Phys. 16, 1284 (1975)

7. Lanford, O.E.: Commun. math. Phys. 11, 257 (1969)

8. Sinai, Ya. G.: Vestn. Markov Univ., Ser. I, Mat. Meh. 29, 152 (1974)

9. Marchioro, C., Pellegrinotti,A., Presutti, E.: Commun. math. Phys. 40, 175-185 (1975)

10. Goldstein, S., Lebowitz, J. L. Aizenman, M.: In Ref. 2, discuss ergodic properties of infinite classical systems. See also Gurevich, B. M., Sukhov, Ju. M.: Stationary Solutions of the Bogoliubov Hierarchy Equations in Classical Statistical Mechanics, 1. (preprint). In this work, received after the present paper was completed, it is shown that for some type of interactions and some assumptions on $\omega$ a stationary state has to be Gibbs

11. Haag, R., Kastler, D., Trych-Pohlmeyer, E.: Commun. math. Phys. 38, 173 (1974)

12. Kastler, D.: Equilibrium states of matter and operator algebras, Proc. of the Roma Conf. on $C^{*}$-Algebras, Inst. Nationale Alta Mat. 1975, to appear

13. Bratteli, O, Kastler, D.: Commun. math. Phys. 46, 37-42 (1976)

14. Haag, R., Kastler, D.: Stability and equilibrium states II, to appear

15. Gallavotti, G., Verboven,E.: Nuevo Cimento 28 B, 274 (1975). For further investigations of the classical KMS condition see Gallavotti, G., Pulvirenti, E.: Commun. math. Phys. 46, 1-9 (1976)

16. Goldstein, S., Aizenman, M., Lebowitz, J. L. : to appear

17. Aizenman, M.: Thesis, Yeshiva University (1975)

18. Gallavotti, G. : Preprint for the series in honor of D. Graffi, in print

Communicated by K. Hepp

Received June 10, 1975 